\title{
PARENTAL PERCEPTIONS OF CHARACTERISTICS AND OUTCOMES OF CHILDREN AND FAMILIES IN THE PRETORIA COCHLEAR IMPLANT PROGRAMME
}

\author{
Marguerite Jessop, Alta Kritzinger and Nellie Venter
}

\author{
Department of Communication Pathology \\ University of Pretoria
}

\begin{abstract}
In order to address the Pretoria Cochlear Implant Programme's (PCIP) need for an in-depth analysis of the variables related to outcomes of children with cochlear implants and their families, a comprehensive postal questionnaire was used in a cross-sectional study employing quantitative methods. The aim was to determine the perceptions of parents/caregivers of children with cochlear implants in the PCIP of the children's outcomes, and to collate relevant data pertaining to 45 children and their families. Results indicated that several key factors played a role in determining a positive outcome in the children's audiological, linguistic, social and educational functioning leading to placement in an inclusive educational setting. The most important indicators were: early age at diagnosis, early intervention with hearing aids and early cochlear implantation. The importance of early intervention is emphasized. The study was a first attempt to document the outcomes of the PCIP, indicating a need to establish a computer database to store data of all clients, which can be used for research and regular programme audits.
\end{abstract}

Keywords: Cochlear implant programme, outcomes, early intervention, variables affecting success, inclusive education, specialized education

\section{INTRODUCTION}

Since the advent of cochlear implantation in children in the mid 1980 's, remarkable progress has been made in the technology of the device and its speech processor, in surgical techniques and in intervention programmes (Clark, Cowan \& Dowell, 1997). Infants are now being implanted at increasingly younger ages (Wright, Purcell \& Reed, 2002). This places a paediatric cochlear implant programme firmly within the realm of early communication intervention (ECI) services. Thus, in such a programme as with all ECI programmes, families are of the utmost importance as many biological and environmental factors play a role in the child's progress (Rossetti, 2001). A major goal of ECI is early identification of risk factors for communication delay, including hearing impairment. There is a worldwide trend toward early identification of hearing loss in infants and children (Wright et al., 2002). Families and their children are therefore entering cochlear implant programmes earlier. In order to best utilise this time advantage (Mayne, Yoshinaga-Itano \& Sedey, 2000), it is necessary that cochlear assessment protocols be fast, efficient and family-centred and that motivation for funding be handled quickly and effectively to decrease the time delay between the family's initial contact with the cochlear team and approval for a cochlear implant, and between approval and implantation. The best possible early rehabilitation services must then follow, therefore opening the possibility for placement of the child in inclusive education instead of special education.

The outcomes of an intervention such as a paediatric cochlear implantation are largely dependent on the family context in which the child finds him/herself (Rossetti, 2001). The selection criteria for implantation of children take into consideration the child's support network (Katz, Burkard \& Medwetsky, 2002), thus ruling out many children who would not have derived benefit from the implant due to a lack of follow-up support. Once enrolled in the cochlear implant programme, however, it is imperative that the family be kept involved throughout the entire process.

Rossetti (2001) supports the idea of utilising parental report and of viewing the parents as accurate sources of information. This approach is in accordance with the notion of fam- ily centered assessment, which serves as a tool to include the family as team members, rally their co-operation, empower them as informed decision makers and thereby ensure their continued involvement in the programme. Increased levels of parental involvement also serve to enable parents to better fulfill their role as advocates for their children (Moog, 2002).

The Pretoria Cochlear Implant Programme (PCIP) had been operating for over a decade, and thus the gathering of descriptive data regarding this rapidly growing and changing population of patients was imperative for ongoing research, information sharing and efficacy of service delivery to families. There is increased pressure for outcome studies, or programme audits, to justify the cost of cochlear implantation and to demonstrate accountability for this cost in terms of efficacy (Summerfield \& Marshall, 1999). If there is to be state subsidising of cochlear implants in South Africa for those children whose parents do not have the means to fund the procedure privately, strong motivation needs to come from sound research on the efficacy and cost benefits of cochlear implants for children who are profoundly deaf. In order to describe the outcomes of the programme, a programme audit was required. Since existing data were incomplete, the problem statement for the present study arose directly from this need. While both measured and perceived outcomes should be described for a complete database of a cochlear implant programme, the present study was undertaken with the focus on a family-centred approach and for this reason, outcomes as perceived by parents were described. In order to conduct a study involving outcomes of children with cochlear implants, descriptive data on the child as well as his/her context were required.

As demonstrated in the outcomes reports of the Nottingham (Outcomes Report, 1990-2000) and Birmingham Paediatric Cochear Implant Programmes (Outcomes Report, 1997), a revision and a constant shifting of the boundaries of selection criteria for paediatric cochlear implantation have accompanied the developments in technology and the decreasing age of implantation (Katz et al., 2002). Since the South African context is unique, in terms of population characteristics and the fact that no state funding is available for cochlear implants, local research within its cochlear implant programmes, it is necessary for programmes to revise their own selection criteria. 
Since selection criteria ultimately dictate the profile of a programme's clients, it follows that careful review of both a programme's outcomes and selection criteria are required to build an accurate picture of the clients in that programme (Chester-Brown, 2005). In addition, knowledge of the variables surrounding a child with a cochlear implant and his/her family, which either contribute to or hinder success, can provide guidelines for the adaptation of selection criteria to the population potentially being served by a programme.

As information is the key to making informed decisions, it follows that a paediatric cochlear implant programme, which makes life-changing decisions regarding a child's future, requires detailed information about its clients. It followed that a standard system needed to be developed for the PCIP, which could address the need for retrospective data collection to complete case histories of children implanted for the past ten years, as well as to record prospective data on current and new clients. It was anticipated that a protocol firmly grounded in current research would fulfill the programme's information needs as trends move toward earlier implantation, new surgical techniques, more sophisticated technology, changing selection criteria and the pressure for regular outcome studies (Wright et al., 2002). It was further envisaged that analysis of the data might reveal trends that would assist the programme's team in planning more efficiently for the changing profile of patients. The aim of the study was therefore to describe the defining characteristics and perceived outcomes of children and their families enrolled at this programme.

Throughout the literature on paediatric cochlear implantation, it is evident that the variables affecting the success of a child's use of this technology are numerous and complex, and of a highly individual nature. As greater volumes of data become available and early paediatric implantees grow older and can be tracked, the profile of clients' shifts and variables are better understood. Figure 1 summarises and illustrates some of the factors explored by the study.

\section{Variables affecting the success of a child with a cochlear implant}

1. Age at identification of hearing loss and age at onset of hearing loss

2. Age at implantation and experience with the cochlear implant

3. Cause of hearing loss

4. Biological and medical factors

5. Environmental factors: Socio-economic status, cultural variables and parental education

6. Variables related to hearing and auditory system structures

7. Device variables and programme variables

8. Mode of communication

\section{Outcomes of child with cochlear implant}

1. Language acquisition, speech perception and production

2. Academic success, later employment and independence

3. Educational placement

4. Socialization

5. Family dynamics

\section{Outcomes of cochlear programme}

1. Cost effectiveness and funding
2. Programme evaluation
3. Relevance to South African context
4. Shifting selection criteria
5. Protocol revision
6. Research and future planning through tracking of patient profiles
7. Comparison and communication with other programmes

Conceptualised from: Archbold, Nikolopolous, Tait, O’Donaghue, Lutman \& Gregory (2000); Clark et al. (1997); Merenstein \& Gardner (1998); Summerfield \& Marshall (1999).

Figure 1: Summary of factors affecting client and programme outcomes.

\section{METHOD}

\section{Aims}

The main aim of the study was to formulate a description of defining characteristics and outcomes pertaining to the children with cochlear implants (16 years of age and younger) in the PCIP and their families, as perceived by their parents/caregivers. The four sub-aims involved:

- A description of the children's pre- and perinatal, medical, developmental, audiological and educational histories;

- The compilation of a descriptive profile of the families of children with cochlear implants;

- A description of the children's current outcomes as perceived by their parents in terms of audiological, communicative and educational functioning with the cochlear implant;

- The identification of variables affecting the success of the child with a cochlear implant in the PCIP.

\section{Research Design}

The study took the form of a descriptive survey, as it involved acquiring information about the cochlear implantees and their families, by asking the parents questions and tabulating their answers (Leedy \& Ormrod, 2005). The study was crosssectional as it provided descriptive data at one fixed point in time; however, much of the information was attained retrospectively. Since the study involved mainly discrete data, from a fixed set of questions, a quantitative approach was followed. The study was non-experimental, and no variables were manipulated, thus no dependent and independent variables were incorporated (Leedy \& Ormrod, 2005) and no causal relationships were explored

\section{Ethical Considerations}

The research proposal and proposed questionnaire were submitted to the Research Proposal and Ethics Committee of the Faculty of Humanities, University of Pretoria, as well as to the Head of the PCIP. Permission was obtained from the ear, nose and throat specialist in charge of the programme, to collect and use the participants' data for research purposes. His conditions as well as those of the ethics committee were taken into account before undertaking the study. Participants were required to sign and return a consent form accompanying the letter of consent and questionnaire (Strydom, 2002).

\section{Participants}

The target group was the children enrolled in the PCIP and their families. Non-probability convenience sampling was used in participant selection, which precluded generalisation of the results of the survey to the wider population of children enrolled in Cochlear Implant Programmes in South Africa. 
The selection criteria required that the participants had to be parents or caregivers whose children had undergone cochlear implantation, were not older than 16 years of age, and were enrolled in the PCIP.

The register containing the names and telephone numbers of the children enrolled in the PCIP was requested, and parents were then telephonically contacted to determine willingness to participate in the study. Only 71 of the 73 parents and children enrolled in the programme qualified as participants in the study, since two families resided in remote geographic locations and were inaccessible by post. Of the 71 questionnaires that were sent out, 45 were returned, representing a $63.4 \%$ response rate. According to Edwards et al. (2002), a response rate of over $60 \%$ is considered acceptable in a study involving postal questionnaires.

\section{Description of participants}

In general, the majority of the participants' children in the PCIP were white (91\%), Afrikaans speaking (73\%) children representing both genders ( $49 \%$ female and $51 \%$ male), living with both parents $(76 \%)$ and having at least one sibling $(73 \%)$. The children were more often the youngest children in the family $(64 \%)$, and spent the majority of their time with their mothers $(65 \%)$. Two languages were spoken in the homes (with the exception of one home in which three languages were spoken). Children were between 2 and 16 years of age, and were likely to fall in the categories 2-6 years (pre-school), 7-12 years (primary school) or 13-16 years (high school). A wide distribution of ages was thus seen in the participants. Regarding the families, the majority of parents were employed, with fathers/paternal caregivers employed in all families except one. The majority of the families lived in Gauteng (86\%), and was thus relatively close to the PCIP for access to services. The participants of the questionnaire were the children's biological mothers in all cases except one, a mother of two children with cochlear implants in the PCIP, where the child's grandmother was the participant in consultation with the mother.

\section{Materials}

The aims of the study as well as the time and financial constraints in the particular setting underscored the need for a data collection instrument that could collect large volumes of quantitative data from a large number of participants who were spread over a wide geographic area, in the shortest time span and the most standardised way possible (Leedy \& Ormrod, 2005). A postal questionnaire was therefore selected as the data collection instrument.

The researcher compiled the parent questionnaire, with input from other members of the PCIP team. The questionnaire consisted of discrete items and was divided into five sections covering the areas of biographical information, prenatal and birth history, medical history, developmental history, family history, audiological history and current functioning of the child post-implantation. The questionnaire was available in both English and Afrikaans, depending on the language preference of the participant. Since the questionnaire was extensive, closed type questions were chosen for ease and speed of answering. Along with the questionnaire, parents received a covering letter outlining the aims of the study and the possible value thereof, as well as a consent form to sign.

\section{Content and compilation of the questionnaire}

The questionnaire's content was compiled according to each sub-aim using various sources from various fields. The questions in the tool were drawn questions from the CHRIB case history form (Kritzinger \& Louw, 2000) used by the Centre for Early Intervention in Communication Pathology at the University of Pretoria, as well as other questionnaires used at the Department of Communication Pathology, University of Pretoria. Furthermore, the questions and justification for questions were influenced by various sources in the fields of ECI (Rossetti, 2001), neonatal intensive care (Merenstein \& Gardner, 1998), paediatric cochlear implants (Clark et al., 1997), assessment in childhood communication disorders including hearing impairment (Katz et al., 2002), and family assessment (Rossetti, 2001). The format of the database being compiled by the Tygerberg Cochlear Implant Programme was consulted for comparative and evaluative purposes, and the questionnaire was adapted to include information in this tool. Only questions that could be answered by the parents were asked.

Structure of the questionnaire

The first section of the questionnaire involved biographical information pertaining to the child's family circumstance. Questions were chosen to render information about the socio-economic status of the family and the level of education of the people providing stimulation to the child (Dollaghan et al., 1999), as well as the family structure and languages spoken. These questions, as well as those in the next four sections, were used to fulfill the need for information regarding the factors influencing the success of a child with a cochlear implant, visually depicted in the upper section in Figure 1.

The next section, covering the history of pregnancy and birth history, was included since many causes of hearing impairment have their origin in the prenatal period or with birth trauma (Merenstein \& Gardner, 1998; Rossetti, 2001). More specific, closed-ended questions regarding the child's condition after birth were included in table format to cover all the possible risk factors for hearing loss and developmental delay in this time period (Kritzinger \& Louw, 2000).

The medical history of the child, including hospitalisations as well as a table listing conditions associated with sensorineural hearing loss, additional disabilities and developmental delay influencing success with a cochlear implant, comprised the next section (Blair, Ramey \& Hardin, 1995; Rossetti, 2001).

Thereafter, the developmental history of the child was covered in a table detailing developmental milestones, the development of feeding and parental perceptions of their infants' development prior to the onset of hearing loss. The family history surrounding the child was documented next, with emphasis on the presence of a hereditary component to the child's hearing loss (Angeli et al., 2000; Matsushiro et al., 2002), as well as other disabilities, which may affect progress with a cochlear implant such as a learning disability or genetic syndromes (Pisoni, 2000).

The child's audiological history prior to and after cochlear implantation was then probed followed by the last section of the questionnaire which used categorical type multiple choice questions arranged in tables of options to describe the child's current educational setting, use of his or her cochlear implant, level of communication, audiological development and the level of speech intelligibility attained by the child (Ching, Psarros, Hill, Dillon \& Incerti, 2001). This section related to the perceived outcomes of the child with a cochlear implant depicted in Figure 1. The scales used in the questionnaire for rating audiological development and the child's attained level of speech intelligibility were respectively adapted and included the Lip Profile (Listening Progress Profile) of Environmental Sounds (Archbold, 1993) and the SIR Speech Intelligibility 
Rating Scale (Allen, Nikoloploulos \& O'Donoghue, 1998). Further questioning involved the support services and rehabilitative therapy available to the child, the child's use of a telephone and the ability to enjoy music.

\section{Procedures}

\section{Reliability and validity}

A thorough literature review served as underpinning for the questionnaire. The questionnaire was structured in a logical way, using simple response choices, easily answerable questions and a user-friendly format. A pilot study was conducted in order to increase the reliability and validity of the study.

\section{Pilot study}

The aims of the pilot study were refinement and adjustment of the structure and content of the questionnaire based on pre-testing (Leedy \& Ormrod, 2005). The participant was the biological mother of one child with a cochlear implant, who was slightly above the maximum age defined for participant selection in this study. The participant of the pilot study was therefore not utilised in the main study. The participant was given the choice to participate in the pilot study of her own free will and complied with all participant selection criteria set for the main study, besides the age of the child. Valuable comments were made by the participant, which aided refinement of the questionnaire for the optimal collection of accurate information for the study. Since only one participant was utilised in the pilot study, it is recognized as a weakness.

\section{Data collection procedures}

Data were collected via a comprehensive questionnaire filled in by the parents/caregivers of the children in the PCIP. Questionnaires were posted to all families as well as postagepaid, return-addressed envelopes and informed consent letters. Prior to posting of questionnaires, the families were contacted telephonically. This initial phone call served as a means to explain the nature of the study and its ethical issues, request permission to use data and determine willingness to participate in the study. Follow up phone calls three weeks after posting the questionnaires served as reminders to those respondents who had not yet returned their questionnaires. No further reminders were given, since it may have violated the ethical principle of voluntary participation.

\section{Data recording/preparation and analysis procedures}

Data were organised using manual coding strategies for each answer to questionnaire items, after which it was entered into a Microsoft Excel spreadsheet. A computerised system for the analysis of descriptive statistics, namely the $S A S(\mathbb{B})$ (Statistical Analysis System, 1999) was used to provide frequency and percentage information. Both dichotomous and categorical nominal, as well as ordinal and numerical measurement scales were used due to the variety of information being gathered. For the same reason, both measures of central tendency and of dispersion were used to provide the rich description requirèd by the data.

\section{RESULTS AND DISCUSSION}

The findings of the study are presented and discussed according to the sub-aims. Where appropriate, the sample was divided into two groups, namely those participants attending specialised schools and those in inclusive educational (IE) settings, and a comparison was made between the groups. This was used to aid the search for variables that could contribute to the success of a child with a cochlear implant in terms of the auditory, linguistic, everyday functioning and academic skills considered by parents and professionals when deciding on the choice of educational placement. As summarised in Figure 1, a number of audiological, biological and environmental factors affect the outcomes of a child with a cochlear implant, and the family unit is the key to understanding the development of the child. For this reason the variables relating to the families of participants were discussed, in addition to variables directly related to the children in the study. The outcomes of children, as measured by parental report, were presented and expanded on by comparison to outcomes of other programmes, and by linking these outcomes to various possible contributing factors in the children's histories. The complete description of the results of the study may be found in Jessop (2005).

Results for sub-aim 1: A description of the prenatal, perinatal, medical, developmental, Audiological and educational histories of the participants' children

\section{Cause of hearing impairment}

According to Table 1 the largest group, namely those in which the cause was unknown to the parents, is of particular interest since this may in part represent the non-syndromic genetic mutations (BJB2 gene) as described in the literature as being frequent in the Caucasian population (Matsushiro et al., 2002). Angeli et al. (2000) report that there are at least 20 different mutations of this gene, caused by hereditary or disease processes; and that the numbers of these children in cochlear implant programmes warrant standard screening for this aetiology. The Joint Committee on Infant Hearing (JCIH, 2000) stated that, in the near future, advances in knowledge regarding recessive genes responsible for non-syndromic hearing impairment could dramatically reduce the number of children whose aetiology of hearing impairment is unknown.

Table 1. Causes of hearing loss in children ( $\mathrm{N}=45)$

\begin{tabular}{|l|c|}
\hline \multicolumn{1}{|c|}{ Cause of hearing loss } & $\begin{array}{c}\text { Percentage } \\
\text { of children (\%) }\end{array}$ \\
\hline Unknown & $17(38 \%)$ \\
\hline Meningitis & $4(9 \%)$ \\
\hline $\begin{array}{l}\text { Genetic syndrome associated with hearing impair- } \\
\text { ment }\end{array}$ & $4(9 \%)$ \\
\hline Prenatal complications & $3(7 \%)$ \\
\hline Birth trauma (foetal distress, asphyxia) & $3(7 \%)$ \\
\hline Hereditary hearing impairment & $3(7 \%)$ \\
\hline Congenital Rubella syndrome & $3(7 \%)$ \\
\hline Maternal cytomegalovirus infection & $2(4 \%)$ \\
\hline Viral infection & $1(2 \%)$ \\
\hline Kidney disorder & $1(2 \%)$ \\
\hline Chronic otitis media & $1(2 \%)$ \\
\hline Ototoxic medication & $1(2 \%)$ \\
\hline Medication ingested by mother whilst pregnant & $1(2 \%)$ \\
\hline Myelin sheath disorder & $1(2 \%)$ \\
\hline ToTAL & $\mathbf{4 5 ( 1 0 0 \% )}$ \\
\hline
\end{tabular}




\section{Nature of the hearing loss}

Only $16 \%$ of children in the current study had an acquired hearing impairment, while the hearing impairment was congenital in $84 \%$ of cases. Data revealed that $28 \%$ of children (including those with congenital and acquired hearing impairments) reported a progressive hearing loss, and in $7 \%$ of cases this was of a fluctuating nature. The reasons for the progression were the presence of Reno tubular Fanconi syndrome, hereditary hearing loss, unknown causes and CMV. The majority of children, with the exception of those affected by meningitis and perinatal factors, had congenital hearing impairments, which emphasizes age at implantation as a crucial factor in language development. Of the 7 children (16\%) in the sample whose hearing loss was acquired, $85 \%$ of parents reported that development prior to the onset of hearing loss was normal, indicating the absence of early developing additional conditions. Children with acquired hearing loss have the advantage of early exposure to sound and possibly some linguistic development, and generally develop language at a faster rate post-implant than children with congenital hearing loss (Katz et al., 2002). These characteristics of the children added to the complexity and heterogeneity of the data, and imply an additional dimension to the planning, expectations and outcomes of cochlear implant programmes.

\section{Biological and medical variables}

The medical histories of the participants' children with cochlear implants were compared when they were divided into two distinct groups, i.e. those in inclusive education (IE), and those in specialised education (SE). The only significant difference highlighted by this comparison was the greater number of children in the SE group who suffered from ADD/ ADHD (reported by parents), namely $5 \%$ of the IE group as opposed to $21 \%$ of the SE group. This finding corresponds with that of Pisoni (2000), calling for more emphasis in cochlear implant research on issues of information processing such as attention; memory; perception and learning to explain the individual differences in outcome after paediatric cochlear implantation. The group in IE also seemed to suffer from fewer bouts of general childhood illnesses such as measles, mumps and chicken pox that could be interpreted to indicate a generally higher resistance to infectious illness and possibly better general immunity and health leading to fewer absences from school and missed learning opportunities.

Both groups indicated that middle ear infections as well as upper respiratory tract infections were frequent occurrences, which would have a temporary negative impact on auditory functioning. The $\mathrm{JCIH}(2000)$ reported otitis media to have an especially negative effect in children with sensorineural hearing loss. None of the children in IE suffered from a chronic illness, as opposed to one participant in the SE group.

The severity of the disorders of vision, not indicated by these broad categories, revealed itself to be significant on closer inspection. The children in inclusive settings had mild visual acuity problems, corrected with lenses,' while those in the second group had more severe visual complications including retinitis pigmentosa.

\section{History of diagnosis of hearing loss and intervention}

Hearing loss was generally suspected by mothers/ parents by 12 months of age (62\%), although $18 \%$ of parents suspected that their infants could not hear by 6 months. All children's hearing losses were suspected by 44 months. In $44 \%$ of cases it was the mother who noticed the child's lack of response to sound, in $42 \%$ of cases both parents, in $9 \%$ of cases the grandparent, in $3 \%$ of cases it was other people, and in only $2 \%$ of children was it a teacher or therapist. Only $4 \%$ of children were diagnosed by 6 months, a figure which lends support to the idea of compulsory hearing screening after birth as implemented in the USA (Mayne et al., 2000). In five cases, it was reported by parents through the addition of information not directly asked in the questionnaire, that doctors did not react immediately to mothers' concerns regarding their children's development, despite findings indicating the high reliability of parental reports of concern (Rossetti, 2001).

There was a substantial delay between three steps: concern, diagnosis and treatment of hearing loss in the participants' children. The $28 \%$ of children with progressive hearing loss, as well as the $7 \%$ whose hearing losses fluctuated, may have contributed to the delays. A shorter time delay between the three steps in the process prior to cochlear implantation would necessarily imply a younger age of implantation and should be a goal of healthcare professionals (Mayne et al., 2000) and the health system in South Africa.

Figure 2 depicts the length of time that the child wore hearing aids, implying the time between fitting of hearing aids and cochlear implantation.

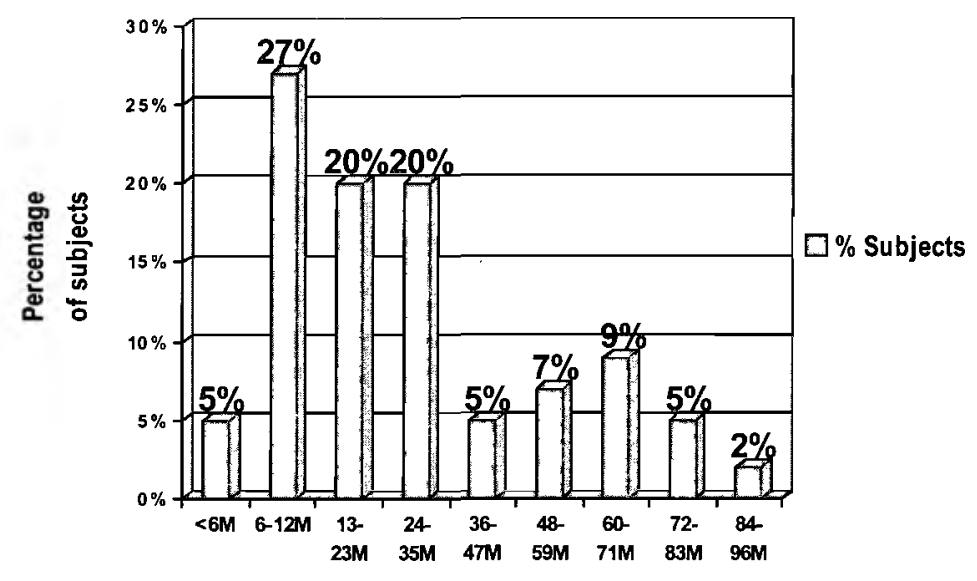

Ages of subjects

Figure 2: Period for which children wore hearing aids prior to cochlear implantation.

From Figure 2 it can be seen that, while the required period for wearing hearing aids prior to establishing candidacy for cochlear implantation was 6 months, most participants' children wore hearing aids for longer than this period prior to cochlear implantation. It is an important requirement of the PCIP that while the child is wearing hearing aids during the trial period, he/she should either be included in a specialised classroom or receive intensive, appropriate auditory stimulation. The highest percentage of children $(27 \%)$ wore hearing aids for between 6 and 12 months, with a large percentage $(40 \%)$ wearing hearing aids for 13-36 months. These children received little or no benefit from their hearing aids in this time, as this is a candidacy criterion for cochlear implantation.

Several factors may play a role in affecting the duration of hearing aid use prior to cochlear implantation, such as late referral to the cochlear implant programme, difficulty obtaining the necessary funding for the cochlear implantation, delays in the process of making the decision to operate as well as in the process of establishing candidacy, and the health of the child in order to make the surgery viable. All of these variables may play a greater or 
lesser role in determining the speed with which a child is implanted, although in the PCIP delays are not generated by an annual quota of cochlear implants or a lengthy waiting list as it is largely still an elective procedure and thus part of the private health care sector.

Funding can pose a significant barrier to early implantation for many South African children. Of the 45 children, only one received a small amount of state funding for the procedure. In $84 \%$ of cases the family's medical aid scheme paid for the implantation, and some cases were funded privately by the family and through donations. This implies a waiting period before cochlear implantation as many families raise the necessary funds themselves.

\section{Age at cochlear implantation}

The distribution of ages at which children in the study received their cochlear implants was between 12 months and 10 years. The largest groups, in descending order, were those implanted at age 2-3, age 1-2, and age 3-4 years respectively. A world-wide trend toward earlier cochlear implantation exists (Archbold et al., 2000). Results therefore indicated that the PCIP data were in accordance with this trend, with more children being implanted prior to age 5 years than later.

\section{Audiological variables: Side of implant and reasons}

In most children $(60 \%)$ the right side was chosen for cochlear implantation, while $36 \%$ had their left ear implanted and $4 \%$ received bilateral implants. When the hearing loss was similar in both ears, the dexterity of the child was taken into account by the surgeon. As most people are right handed, this could explain the high percentage of right side implants.

\section{Reasons for choice of side for cochlear implantation}

The largest group of participants $(38 \%)$ chose the child's poorer ear (with less residual hearing), with handedness, most normally formed cochlea and hearing nerve, ear which had the most stimulation and thus had the most residual hearing, frequent middle ear infections on one side, facial nerve implications or the child's own choice as the other reasons for choice of cochlear implantation. Side of implant may be affected by many factors where there is not a clear structural or surgical choice of side. As selection criteria shift to include children with greater levels of residual hearing, this decision becomes more complex as the possibility of 'losing' the residual hearing in the implanted ear due to the effects of surgery, including cochlear trauma and connective tissue growth, become relevant (Kiefer et al., 1998). In general, the side with the least residual hearing is then implanted, affording two advantages to the child and the family, namely the psychological sense of security that his/her hearing sense will not be obliterated completely, as well as the possibility of making use of the binaural/bimodal advantage with a well fitted hearing aid on the non-implanted ear. Philosophies and viewpoints regarding the choice of ear to be implanted have changed in the PCIP as a function of time, new research findings, shifting selection criteria and changing surgical techniques (Katz et al., 2002). As a rule, the largest number of participants in the current study had chosen their children's 'weaker' ear for implantation. This raises the next issue, namely the use of a hearing aid on the non -implanted side.

\section{Hearing aid use on non-implanted ear}

Only $44 \%$ of children wore a hearing aid on the contra lateral ear. Reasons given for not wearing a hearing aid in- cluded the fact that the ear had too little residual hearing to benefit, not knowing that it was advisable, and the child's refusal to wear the hearing aid. The reasons for wearing the hearing aid included better sound, better localization, stimulation of the acoustic nerve, the school's policy, more balanced hearing, making use of residual hearing, and recommendation by the audiologist. In Pretoria, the majority of specialised schools advocate the wearing of a hearing aid on the nonimplanted side, at least during school hours. This, together with the audiologists of the PCIP's own frequent recommendations to encourage this practice, may have accounted for a large percentage of the participants' motivation to do so, although this is a fairly recent development based on more recent research and the advent of bilateral cochlear implants as a viable option. Studies demonstrating the advantages of bilateral implants are growing in number (Ertmer, 2002). In the PCIP only two children had received bilateral cochlear implants, both in successive operations as opposed to simultaneous operations. According to the parents, of the two, one wears both his devices well and the other refuses to wear the second device.

\section{Results for sub-aim 2: Compilation of a descriptive profile of the families of children with cochlear implants in the PCIP}

In this section a profile was created of the families of children with cochlear implants in the study was created, including information on the prevalence of hearing loss or additional disorders of speech, language or learning in families and educational level and employment status of parents. In eight children's families there existed a history of childhood hearing loss. Participants were further asked to report on a family history of speech/language or academic difficulties not related to hearing loss. In the SE group, $17 \%$ of families had a history of learning/academic and serious speech/language difficulties in childhood. This was higher than the $5 \%$ reported in the IE group, and may have been a factor contributing to the need for SE. The presence of these difficulties in the parent might have affected their level of stimulation of the child (Dollaghan et al., 1999), while the presence of these hereditary traits in the children themselves could have hampered their success in acquiring language and academic progress.

Environmental variables related to the family unit were reported as parental education and employment status in order to give an indication of socio-economic status and the amount of time spent at home with the children by the maternal and paternal parents/caregivers respectively. It followed that the parent spending the most time with the children would play a greater role in academic development, and this parent's own educational status might then be of greater relevance. The results showed that mothers/maternal caregivers of the children in IE had a higher level of educational status in general. The most pronounced differences were seen in the highest level, namely university degree that included $26 \%$ of mothers in the IE group and only $8 \%$ of mothers in the SE group. The lowest level measured, namely standard 8 , included only $5 \%$ of mothers of children in inclusive settings and $17 \%$ of mothers in the $\mathrm{SE}$ group. A similar trend was seen with paternal parents/ caregivers, where $37 \%$ of fathers of children in IE had a university degree as opposed to $23 \%$ of fathers of children in specialised settings. Thus it would appear from these data that the level of maternal and paternal educational status might play a role in the academic success of a child with a cochlear implant. 
Results for sub-aim 3: Description of children's current perceived outcomes in terms of auditory, communicative and educational functioning

\section{Auditory outcomes: Use of the device}

All participants reported that the cochlear implant device was worn at all times (all waking hours), at home and at school.

\section{Auditory performance}

Speech was most easily detected and identified by the participants, which was probably due to the cochlear implant's bias toward providing access to sounds in the speech frequency (Gibson, Rennie \& Psarros, 2000). While over 93\% of participants could detect music, their own names and family names, soft sounds and whispered speech, fewer participants could also discriminate these sounds. Access to sound, however, was greatly improved by cochlear implantation.

\section{Linguistic outcomes: Mode of communication}

From the breakdown in Table 2 it was evident that the majority of children was using the auditory-oral mode of communication as opposed to signing, and was in various stages of development of spoken language. The largest number of children was categorized in the 'functional language' column, which in this context meant that they were using connected speech in complete or incomplete sentences more often than 3-word utterances or shorter units of language.

More children in this study were using single words than more complex word combinations, however, an even larger group was using mature sentence forms. This indicated that in the present cross sectional study, more children were in either the earlier, or the more advanced stages of language acquisition than in the middle stages.

This result did not necessarily imply that these children were of a certain age group, since the linguistic outcome of paediatric cochlear implantation is highly variable.

The population is a "notoriously heterogeneous population with great variation in their auditory, cognitive and linguistic maturity that may be impossible to quantify before intervention (O'Donague et al., 2000, p.467), however, duration of devịcé use could play a role.

Table 2: Mode of communication and level of language development for all children $(\mathrm{N}=45)$

\begin{tabular}{|l|c|}
\hline \multicolumn{1}{|c|}{$\begin{array}{c}\text { Mode/level of } \\
\text { communication development }\end{array}$} & $\begin{array}{c}\text { Percentage of } \\
\text { children using } \\
\text { this modellevel } \\
\text { often }\end{array}$ \\
\hline Sounds & $36 \%$ \\
\hline Natural gestures & $38 \%$ \\
\hline Word approximations & $31 \%$ \\
\hline AVERAGE FOR PREVERBAL LEVEL & $\mathbf{3 5 \%}$ \\
\hline Single words & $36 \%$ \\
\hline AVERAGE FOR TRANSITIONAL LEVEL & $\mathbf{3 6 \%}$ \\
\hline TwO word combinations & $29 \%$ \\
\hline Three word combinations & $29 \%$ \\
\hline AVERAGE FOR LEVEL ACQUIRING FUNCTIONAL & $\mathbf{2 9 \%}$ \\
\hline LANGUAGE & $36 \%$ \\
\hline Incomplete sentences & $\mathbf{4 7 \%}$ \\
\hline Complete sentences & $\mathbf{4 2} \%$ \\
\hline SIGN LANGUAGE & $\mathbf{1 1 \%}$ \\
\hline SPEECH PLUS SIGN: TOTAL COMMUNICATION & $\mathbf{2 2 \%}$ \\
\hline
\end{tabular}

A possible cause of the variation in speed and extent of linguistic outcome was the central auditory processing mechanisms that are crucial to the perception of speech through a cochlear implant, and for which there are as yet no reliable measures (O'Donague et al., 2000). From this discussion it is evident that cochlear implantation resulted in access to a wide range of sounds, including speech and soft environmental sounds for most of the children in the PCIP, although children in the IE group showed slightly better outcomes. All the children wore their cochlear implants, and the majority was acquiring, or had acquired, speech through listening.

\section{Educational placement outcomes: Comparison of variables and outcomes for children in $I E$ and those in $S E$ settings.}

For most cochlear implant programmes the goal of early implantation is to allow the child to be placed into an IE setting and to function among his/her normal hearing peers. The Nottingham Paediatric Cochlear Implant Programme (1997) reported placing 53\% of their preschool age children in inclusive settings at two years post-implant, while the Birmingham Paediatric Cochlear Implant Programme (2000) reported placing $17 \%$ of their total population, of all ages, in regular classrooms.

Tables 3 and 4 endeavour to profile various characteristics and outcomes as they pertain to children in IE, and those in SE settings. Two children were at home with their mothers and were not in any educational setting; therefore the total for the two groups was 43 children. Only two children attended schools for the Deaf where sign language was a medium of tuition.

\section{- Age at diagnosis of hearing loss}

The children in the preschool IE group received a diagnosis slightly earlier than those in the SE group, with an average difference of 6 months, as illustrated by Tables 3 and 4 . The same trend was seen in the primary school (average difference 17 months) as well as the high school (average difference 9 months) groups. Since diagnosis is the first step toward amplification and cochlear implantation, it seems that the children in the SE group were, in general, at a slight time disadvantage from the start (Yoshinaga-Itano, Sedey, Coulter \& Mehl, 1998).

\section{- Age at fitting of hearing aids}

In general, children in the three groups in IE were fitted with hearing aids earlier and younger than those in SE settings. The IE group thus had access to sound, prior to cochlear implantation, at a younger age than the SE group, allowing important neurological development to take place in the auditory cortex (Katz et al., 2002).

\section{- Age at cochlear implantation}

The largest group of IE preschoolers was implanted between the ages of 1 and 2, while the majority of SE preschool children received their cochlear implants between the ages of 2 and 3 years of age. This represented a significant difference in the children's groups in terms of neural plasticity and capacity to acquire age-appropriate language, although it is worth noting that the majority of children in this age range were implanted at or before 3 years of age. As seen in Tables 3 and 4, no children were implanted prior to age 3 years in the primary school SE group, and this group received their cochlear implants on average 14 months later than their peers in the IE group. Since age has been identified through-out the literature as the major determining factor in successful paediatric co- 
Table 3: Description of characteristics of children in inclusive educational settings ( $N=19)$

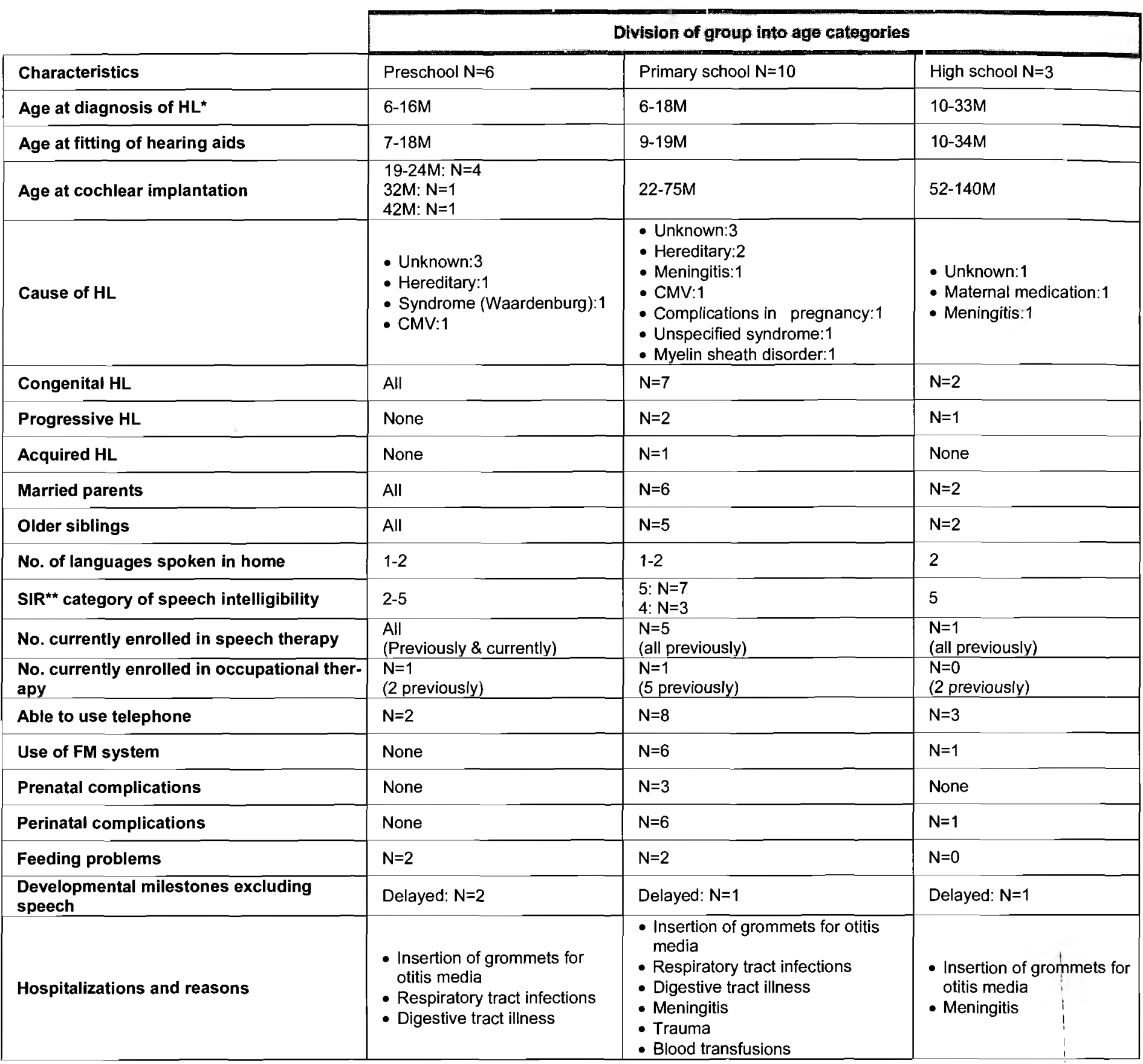

* HL: Hearing loss; ** SIR: Speech Intelligibility Rating Scale

chlear implantation (Mayne et al., 2000), these results point to the urgency for more efficient systems of diagnosis, referral to cochlear implant teams, establishing of candidacy and funding for cochlear implantation in the PCIP.

\section{- Cause of hearing loss}

The etiologies of hearing loss for which the numbers were comparable between the two groups were genetic syndromes and unknown causes. More SE than IE children lost their hearing due to congenital rubella syndrome and birth trauma, while more children in IE had hereditary hearing loss. Children who experienced birth trauma might be expected to have suffered further developmental consequences of neonatal asphyxia (Merenstein \& Gardner, 1998), including increased risk for developmental delays in all areas including sensory, motor, cognitive and speech/language development (Rossetti, 2001). Hereditary, non-syndromic hearing loss is more likely to go unaccompanied by additional disabilities (Angeli et al., 2000; Matsushiro et al., 2002), implying that this specific cause of hearing loss may be related to higher levels of success with a cochlear implant.

- Nature of hearing loss: progressive and congenital hearing loss

In Tables 3 and 4 it can be seen that slightly more children in IE had exposure to sound prior to the onset of their hearing losses, placing them at an advantage in terms of neurological maturation of the auditory cortex, even if exposure to sound was brief (Ponton, Don, Eggermont, Waring \& Masuda, 1996).

\section{- Marital status of parents}

Most of the parents of children with cochlear implants in both groups were married. Most children thus benefited from two parents, nuclear family contexts, found to be positively correlated with significantly better academic and language outcomes (Calderon \& Low, 1998). 
Table 4. Description of children in specialised educational settings $(\mathrm{N}=24)$

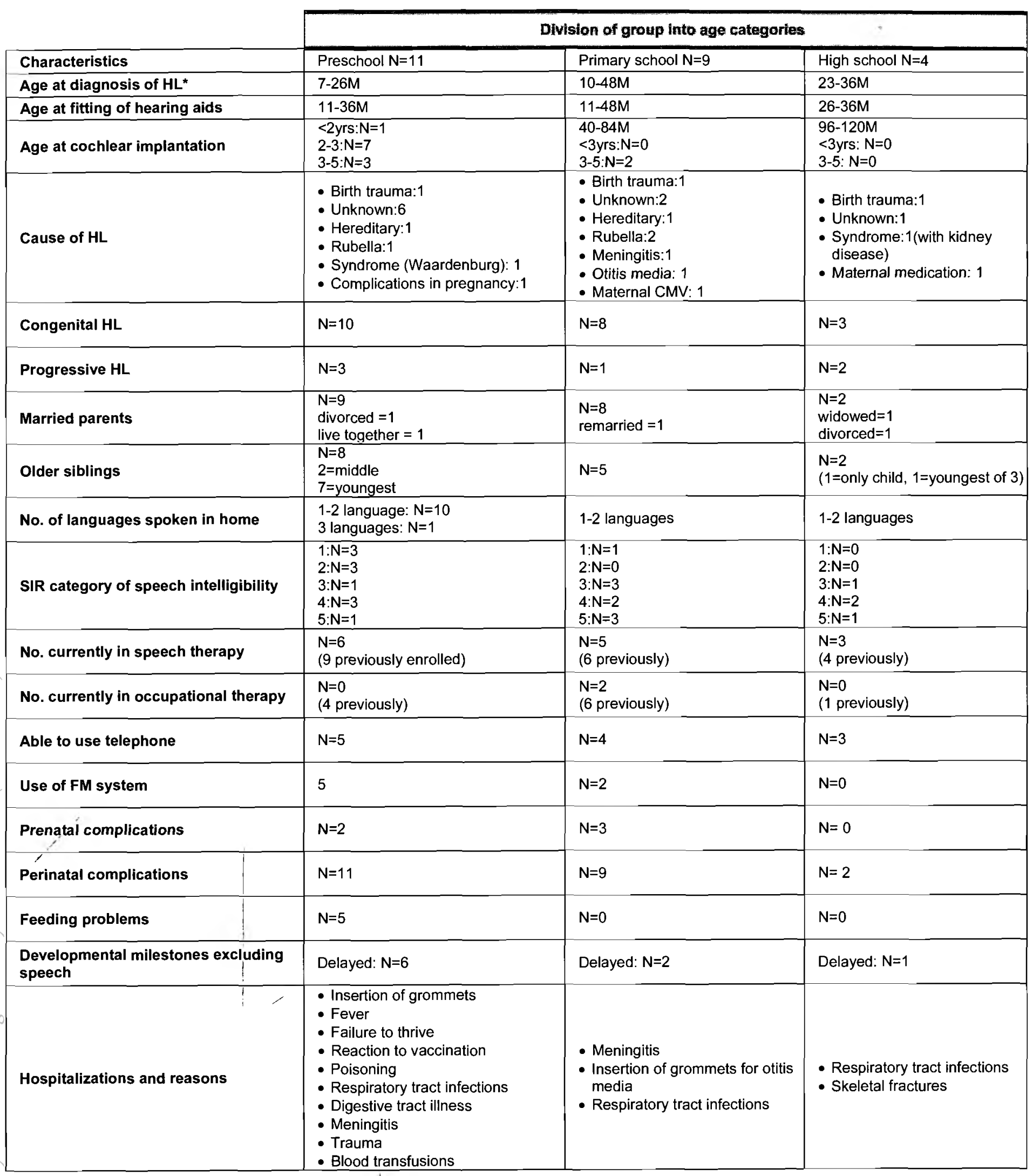

*HL: Hearing loss

\section{- Presence of older siblings}

This variable was explored due to the possible advantages to language acquisition of having an older sibling as a peer language model. Results indicated that slightly more of the children in the IE group had the advantage of an older sibling in the home. In general, most children in this study were the youngest and had older siblings (62-68\%).
- Number of languages spoken in the home

The majority of families in both groups spoke one or two languages in the home. The possible effects of language confusion on the child were not significant in either group, and closer inspection revealed that in both groups, bilingual families attempted to speak only one language in the home to accommodate the participant. 
- Speech intelligibility according to the Speech Intelligibility Rating Scale (SIR)

The rating scale used, namely Speech Intelligibility Rating Scale (Allen et al., 1998) (SIR), grades speech intelligibility in 5 levels as follows: 1 indicates unintelligible speech; 2 indicates that intelligible speech is developing in single words in a known context; 3 means speech is intelligible to a listener who concentrates and lip-reads; 4 indicates speech which is intelligible to a listener without much prior experience of deaf speakers; and 5 means speech is intelligible to all listeners. In general, the SIR level reported was higher for preschool children in IE than in SE, and no children in IE had a rating indicating unintelligible speech. For the primary school children, the difference became more pronounced with children in IE reported as having normal or near-normal speech, as opposed to the SE children whose ratings varied widely and tended to be lower. IE children in high school were all rated at the maximum score as being normally intelligible, whereas their peers in SE were generally rated as being more difficult to understand. This variable showed a marked difference between the two groups, indicating that speech intelligibility is likely to be affected by, and affects, decisions regarding educational placement.

\section{- Speech-language and occupational therapy: children} currently and previously enrolled in therapy

Of the preschoolers depicted in Tables 3 and 4, a larger percentage in IE were enrolled in speech-language therapy than those in SE. This could be due to the greater support given in class by specialised settings, or due to the increased pressure on children in IE to keep up with their normal hearing peers, requiring support services outside the school. However, as the ages of children increased, more children in SE were still making use of the services of a speech-language therapist in primary and high school than those in IE. This appeared to indicate that IE required greater levels of support services initially, but that this tends to decrease as the child gets older and becomes fully integrated into the system.

\section{- Telephone use}

As shown in Tables 3 and 4, in both the primary and high school groups, the vast majority of study children in IE could use a telephone to communicate successfully, while the percentages were smaller for the SE group. This may give an indication of hearing with the cochlear implant, central auditory processing of a less than ideal signal such as that heard over a telephone line as well as reliance on speech reading.

\section{- Use of an FM system at school}

Of the preschool age children, almost half $(45 \%)$ of those in $\mathrm{SE}$ used an FM system at school, while none of the IE group did. However, at primary school age only $22 \%$ of SE children were using an FM system as opposed to $60 \%$ of children in IE, presumably as the listening demands in an inclusive classroom increase in the formal education system whereas more adaptations are made for listening in SE classes. The conclusion can be drawn that FM systems are still important after cochlear implantation in order to optimize the listening environment. This is true especially in inclusive primary school classrooms where no special acoustic adaptations are made, class sizes may be large, and children's auditory processing skills are still developing in order to cope with listening in background noise.
- Biological contributing factors: Prenatal and perinatal complications or conditions

In general, more children in the SE group presented with prenatal or perinatal complications or conditions than children in the IE group: $39 \%$ of the SE group as opposed to $17 \%$ of the IE group (see Tables 3 and 4). Due to their far-reaching implications (Rossetti, 2001), these variables may have played a role in placing the children in the SE group at a developmental disadvantage, which in turn may have resulted in their educational placement in special school settings.

\section{- Feeding difficulties in neonatal and early childhood stage}

According to Tables 3 and 4, the group which reported the largest incidence of feeding difficulties was the preschool SE group, although a small number of children in the IE preschool and primary school group also reported feeding difficulties. Early feeding difficulties are a well-known risk factor for communication delay in children (Rossetti, 2001).

\section{- Developmental milestones (excluding speech and language milestones)}

Significantly more of the preschoolers and primary school children in the SE group were reported to have presented with delays in achieving developmental milestones than their peers in IE. This may indicate the presence of additional delays in development of the children who were subsequently placed in SE settings, in areas not directly related to the hearing loss such as motor, sensory and self-help skill development.

\section{- Hospitalisations}

Reasons for hospitalisation were similar for the SE and IE groups, including both the causes of acquired hearing loss (meningitis) and most frequently illnesses affecting the respiratory and digestive systems.

\section{Results for sub-aim 4: Identification of variables affecting the success of children with cochlear implants in the PCIP}

This sub-aim was fulfilled using the results and discussions arising from the division of the participant group into two groups based on educational outcomes. The purpose to provide a set of variables that could be linked to outcome measures of fully IE placement, which implies positive speech, language and auditory outcomes as demonstrated in the preceding results and discussions. In general it can be seen that, in the children of this study, the variables listed in Table 5 were linked to positive outcomes.

Table 5: Eleven most important variables associated with successful outcomes of children with cochlear implants in the PCIP

1. Early age at diagnosis, intervention with hearing aids, and cochlear implantation

2. Absence of prenatal and perinatal complications

3. Higher level of maternal education

4. Developmental milestones achieved at normal ages

5. Absence of early feeding difficulties

6. Use of an FM system in early schooling (primary school level)

7. Intelligible speech

8. Access to $\mathrm{ECl}$

9. Presence of an older sibling

10. Absence of birth trauma and congenital rubella syndrome as causes of hearing loss. (More successful outcomes when cause of hearing loss was hereditary and non-syndromic, or unknown)

11. Later onset of hearing loss: acquired (few children) 
Variables associated with success with a cochlear implant for children in the PCIP: (as measured by placement in IE settings)

In order to provide clarity and structure, these variables in Table 5 can be used by the PCIPs team of professionals to guide programme planning in terms of selection criteria, intervention decisions and counseling regarding realistic expectations in parents of children with cochlear implants. Each child and family's individual profile of risks, strengths and needs can be ascertained, and planning can be optimised. Early diagnosis, early intervention with hearing aids and early cochlear implantation, also confirmed by other studies (Yoshinaga-Itano, 2000; Yoshinaga-Itano et al., 1998) stand out as the most important variables associated with successful outcomes of children with cochlear implants in the PCIP.

\section{CONCLUSION}

The study produced valuable data on the children in the PCIP in 2004, yet the results represent only $63.4 \%$ of those children and their families. Establishing a computer database to store and retrieve client data continuously for regular programme audits and research is recommended to monitor outcomes and to ensure best practice in the programme. A database provides the opportunity to store data on direct clinical observations and measurements, overcoming the limitations of research on parental perceptions of outcomes - a clear limitation of the present study.

The children of this study, although differing widely in age, educational level and setting, and circumstances leading to cochlear implantation, were not representative of the South African population or the population of South African children with severe to profound bilateral hearing loss as a whole. As cochlear implantation is still an expensive, elective procedure falling almost exclusively in the realm of the private health care sector in this country, this profile of 'clients is unlikely to change until state funding for cochlear implantation is secured. This can only be achieved by the concerted efforts of clinicians and researchers to generate similar studies on which to base strong motivation for cochlear implantation, universal infant hearing screening and follow-up programmes for all South African hospitals and clinics in addition to the use of high risk registers, and ECI (Swanepoel, 2004). The study has provided descriptions and guidelines for translating theory into action to prioritise early intervention for children with hearing loss. Such an effort requires an "unprecedented level of commitment and cooperation by all those involved: state and community agencies, professional groups and practitioners, parents, advocacy organizations, researchers, and institutions of higher education" (Guralnick, 2001: p.18). With the challenge of early identification of hearing loss, access to early intervention services for all, and state funding enabling earlier implantation and the reaching of a more representative section of the population realised, the outcomes of cochlear implantation in South Africa may exceed the most optimistic expectations.

\section{REFERENCES}

Allen, M.C., Nikolopoulos, T. N., \& O’Donoghue, G. M. (1998). Speech intelligibility in children following cochlear implantation (SIR). The American Journal of Otology, 19 (6), 742-746.

Angeli, S., Utrera, R., Dib, S., Chiossone, E., Naranjo, C., Henriquez O., \& Porta, M. (2000). Gene mutations in childhood deafness. Acta Otolaryngology, 120, 133-136.

Archbold, S. (1993). Lip Profile (Listening Progress Profile). Nottingham Paediatric Cochlear Implant Programme, Nottingham.

Archbold, S. M., Nikolopoulos, T. P., Tait, M., O’Donaghue, G.M., Lutman, M.E., \& Gregory, S. (2000). Approach to communication, speech perception and intelligibility after paediatric cochlear implantation. British Journal of Audiology, 34, 257-264.

Birmingham Paediatric Cochlear Implant Programme. (2001). 1990-2000, Unpublished Outcomes Report, NHS Trust, Birmingham Children's Hospital, UK.

Blair, C., Ramey, C. T., \& Hardin, J. M. (1995). Early intervention for low birthweight, premature infants: Participation and intellectual development. American Journal on Mental Retardation, 99(5), 542-554.

Calderon, R., \& Low, S. (1998). Early social-emotional, language, and academic development in children with hearing loss: Families with and without fathers. American Annals of the Deaf, 143, 225-234.

Chester-Brown, R. (2005). Audiological criteria in children and adults. Presentation at the Cochlear Implant Conference: Issues for new populations. 16-19 March 2005. Spier Estate, Stellenbosch, South Africa.

Ching, T. Y. C., Psarros, C., Hill, M., Dillon, H., \& Incerti, P. (2001). Should children who use cochlear implants wear hearing aids in the opposite ear? Ear \& Hearing, 22(5), 365 380

Ching, T. Y. C., Psarros, C., Hill, M., Dillon, H., Incerti, P., \& Hill, M. (2002). Management of children using cochlear implants and hearing aids. The Volta Review, 103(1), 39-57.

Clark, G. M., Cowan, R. S. C., \& Dowell, R. C. (1997). Cochlear implantation for infants and children: Advances. San Diego: Singular Publishing Group.

Dollaghan, C.A., Campbell, T. F., Paradise, J. L., Feldman, H. M., Janosky, J. E., Pitcairn, D. N., \& Kurs-Lasky, M. (1999). Maternal education and measures of early speech and language. Journal of Speech, Language and Hearing Research, 42, 1432-1443.

Edwards, P., Roberts, I., Clarke, M., DiGuiseppi, C., Pratap, S., Wentz, R., \& Kwan, I. (2002). Increasing response rates to postal questionnaires: Systematic review. British Medical Journal, 324, 1183-1185.

Ertmer, D. J. (2002). Technological innovations and intervention practices for children with cochlear implants. Language, Speech and Hearing Services in Schools, 33, 218-221.

Gibson, W., Rennie, M., \& Psarros, C. (2000). Outcome after cochlear implantation and auditory verbal training in terms of speech perception, speech production and language. Advanced Otorhinolaryngology, 57, 251-253.

Guralnick, M. (2001). Developmental systems model for early intervention. Infants and Young Children, 14 (2), 1-18.

Jessop, M. A. (2005). Clients of the Pretoria Cochlear Implant Programme: Characteristics and perceived outcomes of children and their families. Unpublished Masters Dissertation, Department of Communication Pathology, University of Pretoria.

Joint Committee on Infant Hearing. (2000). Year 2000 Position Statement: Principles and guidelines for early detection and intervention programs. American Journal of Audiology, 9, 9-29.

Katz, J., Burkard, R. F., \& Medwetsky, L. (2002). Handbook of clinical audiology. (5 ${ }^{\text {th }}$ ed.). Philadelphia: Lippincott Williams and Wilkins.

Kiefer, J., von Ilberg, C., Reimer, B., Knecht, R., Gall, V., Diller, G., Stürzbecher, E., Pfennigdorff, T., \& Spelsberg, A. (1998). Results of cochlear implantation in patients with severe to profound hearing loss - Implications for patient selection with parents of deaf and hearing children. Audiology, 37, 382-395.

Kritzinger, A.M., \& Louw, B. (2000). A comprehensive assessment protocol for infants and toddlers at risk for communication disorders. Part 1: Case History. Clinica: Applications in Clinical Practice of Communication Pathology. Monograph 5. Department of Communication Pathology, University of Pretoria. 
Leedy, P. D., \& Ormrod, J. E. (2005). Practical research: Planning and design. ( $\delta^{\text {th }}$ ed.). New Jersey: Pearson Educational International.

Matsushiro, N., Doi, K., Fuse, Y., Nagai, K., Yamamoto, K., Iwaki, T., Kawashima, T., Sawada, A., Hibino, H., \& Kubo, T. (2002). Successful cochlear implantation in prelingual profound deafness resulting from the common 233 delC mutation of the GJB2 gene in the Japanese. The Laryngoscope, 112, 255-261.

Mayne, A. M., Yoshinaga-Itano, C., \& Sedey, A. L. (2000). Receptive vocabulary development of infants and toddlers who are deaf or hard of hearing. The Volta Review, 100(5), 29-52.

Merenstein, G.B., \& Gardner, S.L. (1998). Handbook of neonatal intensive care. $\left(4^{\text {th }}\right.$ ed.). St Louis: Mosby.

Moog, J. S. (2002). Changing expectations for children with cochlear implants. International Journal of Paediatric Otorhinolaryngology, 111, $138-142$.

Neuman, W. L. (2000). Social research methods, qualitative and quantitative approaches. $\left(4^{\text {th }}\right.$ ed.). Boston: Allyn \& Bacon.

Nottingham Paediatric Cochlear Implant Programme. (1997). Unpublished Report: Outcomes for paediatric cochlear implantation. Ropewalk House, Nottingham, UK

Pisoni, D. B. (2000). Cognitive factors and cochlear implants: Some thoughts on perception, learning and memory in speech perception. Ear \& Hearing, 21(1), 70-78.
Ponton, C. W., Don, M., Eggermont, J. J., Waring, M. D., \& Masuda, A. (1996) Maturation of human cortical auditory function: Differences between normalhearing children and children with cochlear implants. Ear \& Hearing, 17(5), 430-437.

Rossetti, L. M. (2001). Communication intervention. Birth to three. ( $2^{\text {nd }}$ ed.). Australia: Singular Thomson Learning.

SAS(B) Version 8. (1999). Cary, North Carolina: SAS Institute Inc.

Strydom, H. (2002). Ethical aspects of research in the social sciences and human service professions. In A. S. De Vos (ed.), Research at grass roots. For the social sciences and human service professions. ( $2^{\text {nd }}$ ed.). Pretoria: J.L. van Schaik Publishers.

Summerfield, A. Q., \& Marshall, D. H. (1999). Paediatric cochlear implantation and health-technology assessment. International Journal of Paediatric Otorhinolaryngology, 47, 141-151.

Swanepoel, D.C.D. (2004). Infant hearing screening at maternal and child health clinics in a developing South African community. Unpublished DPhil dissertation. Department of Communication Pathology, University of Pretoria.

Wright, M., Purcell, A., \& Reed, V. A. (2002). Cochlear implants and infants: expectations and outcomes. Annals of Otology, Rhinology \& Laryngology, 189 , $131-137$.

Yoshinaga-Itano, C., Sedey, A. L., Coulter, D. K., \& Mehl, A. L. (1998). The language of early- and later identified children with hearing loss. Pediatrics, 102, 1161-1171. 